Women, Catholicism and Nation: Writings

about the Past by Amateur or Semi-Professional

Spanish Women Historians (1880-1936)

\title{
Mujeres, catolicismo y nación: los escritos sobre el pasado de las historiadoras aficionadas o semiprofesionales de España $(1880-1936)^{\star}$
}

Alejandro Camino

Universidad Autónoma de Madrid

alejandro.camino@uam.es - https://orcid.org/0000-0003-1532-8239

Fecha recepción: 17.07.2020 / Fecha aceptación: 06.12.2020

\section{Resumen}

Durante las últimas décadas del siglo XIX y las primeras del XX, las mujeres españolas, al igual que ocurrió en la mayor parte del mundo, fueron marginadas y relegadas, explícita o implícitamente, a los márgenes de la profesión de historiador. Ante esta situación, muchas optaron por saciar su interés por el pasado investigando y escribiendo sobre temas que estaban alejados de lo considera-

\begin{abstract}
In late 19th and early 20th century Spain, as in most of the rest of the world, women were marginalised and relegated, whether by law or via informal mechanisms, to the edges of the profession of historian. Adapting to this situation, many women directed their research to subjects considered irrelevant according to the professional canon. The main goal of this article is to analyse the work of
\end{abstract}

\footnotetext{
* Este artículo se enmarca en una investigación predoctoral (FPU16/02273) y en el proyecto «Identidades en movimiento. Flujos, circulación y transformaciones culturales en el espacio atlántico (siglos XIX y XX)» (PID2019-106210GB-I00). Quisiera agradecer a Darina Martykánová, Pilar Toboso e Irene Mendoza sus enriquecedores comentarios sobre el texto.
} 
do como relevante por el canon de la profesión. El objetivo del trabajo es conocer sobre qué temas, y con qué objetivos, escribieron sobre el pasado las mujeres católicas y conservadoras en España que pueden denominarse historiadoras aficionadas o semiprofesionales. Es decir, aquellas que no habían recibido una formación específica reglada o que, cuando la tuvieron, no pudieron consagrarse como profesionales ocupando cátedras y otros puestos de historiador en las instituciones académicas.

\section{Palabras clave}

historiografía, profesión, investigación, mujeres, España
Spanish women of Catholic and conservative tendencies who wrote about the past, to outline the subjects they chose to explore and to identify the aims of their work. This analysis focuses specifically on those who neither received formal training as historians nor obtained recognition as such through appointment to chairs at universities or other posts as historians in academic institutions, and who can therefore be considered amateur or semi-professional historians.

\section{Keywords}

Historiography, profession, research, women, Spain 


\section{Introducción y contexto}

Un/a historiador/a profesional es alguien que vive del dinero que cobra por su trabajo a tiempo completo, ya sea porque la institución para la que trabaja le entrega un salario y/o consigue beneficios suficientes para mantenerse por la venta de libros. Esto es relevante no solo porque permite a la persona obtener el sustento material necesario para su supervivencia (salvo cuando proviene de las clases altas y no necesita trabajar para mantenerse), sino porque, a su vez, la legitiman de cara al exterior como una persona experta en la materia ${ }^{1}$. Para las mujeres, la primera de las posibilidades estuvo muy limitada a finales del siglo XIX y principios del XX por la exclusión que, de forma más o menos explícita, sufrían en la legislación de los diferentes países, mientras que la segunda de las opciones, que ofreció una posibilidad de desarrollo a algunas mujeres en lugares como Inglaterra, en España no fue muy aprovechada.

Como historiador/a semiprofesional considero a quienes intentaron convertirse en profesionales, y se formaron para ello, pero se quedaron a medio camino. En el caso de las mujeres, esta situación fue bastante frecuente en el caso español, sobre todo porque algunas prometedoras investigadoras que se formaron como historiadoras, al constatar la dificultad para acceder a un puesto como profesoras universitarias, optaron por trabajar en el campo archivístico o de la enseñanza de menores, pero sin descuidar su faceta investigadora. Un caso diferente fue el de los/las historiadores/as amateurs o aficionados/as, que son quienes, sin haber recibido una formación académica reglada ni haber tenido la intención de profesionalizarse, debido a su interés por el pasado realizaron investigaciones sobre ello. En cualquier caso, las categorías de profesional, semiprofesional y aficionado no tienen fronteras claras.

En España, fue en la década de 1840 cuando sus elites se hicieron historiadores, en masculino no genérico, y la historia empezó a ser entendida como una ciencia que debía

1. Para la noción de la profesionalización: H. Perkin, The Rise of Professional Society. England Since 1880, Londres, 1989. 
convertirse en un componente de la socialización y de la identificación nacional del futuro ciudadano $^{2}$. No obstante, fue en el periodo finisecular cuando, en una cronología similar a la experimentada en otros países europeos y americanos, comenzó la configuración de la profesionalización historiográfica y de la profesión de historiador. De hecho, fue en 1900 cuando se creó en la Universidad Central un programa de doctorado en historia. Este proceso permitió a los catedráticos novecentistas hacerse con el control del conocimiento histórico, delimitar las fronteras de la llamada «historia científica» y convertir en las instituciones pautadoras de la investigación y de la enseñanza de la historia a la universidad y a la Junta para la Ampliación de Estudios (JAE) ${ }^{3}$.

El ascenso y consolidación de un modelo histórico «científico», durante el siglo XIX y las primeras décadas del XX tuvo fuertes implicaciones para las mujeres a nivel global. Los seminarios, los archivos y las bibliotecas, que fueron los espacios en los que se construyeron y se desarrollaron los métodos para hacer historia profesional, quedaron constituidos como entornos reservados fundamentalmente (o exclusivamente, según el territorio) para los hombres $^{4}$. De esta manera, salvo en casos excepcionales en aquellos países donde las mujeres de clase media-alta y alta tenían un marco ligeramente más propicio para su desarrollo personal en la universidad, como Estados Unidos, Gran Bretaña o los países nórdicos, estas estuvieron apartadas del proceso de profesionalización de la historiografía ${ }^{5}$. Por ejemplo, en Suecia, Di-

2. I. Peiró, Los guardianes de la Historia. La historiografía académica de la Restauración, Zaragoza, 1995; R. Cuesta, Clío en las aulas. La enseñanza de la historia en España entre reformas, ilusiones y rutinas, Madrid, 1998; A. Rivière, Historia, historiadores e historiografía en la Facultad de Letras de la Universidad de Madrid (1843-1868), Madrid, 2001; C. P. Boyd, Historia Patria: Politics, History, and National Identity in Spain, 1875-1975, Nueva York, 1997.

3. G. Pasamar e I. Peiró, Diccionario Akal de historiadores españoles contemporáneos (1840-1980), Madrid, 2002, 12; E. Hernández Sandoica y J. L. Peset, Universidad, poder académico y cambio social (Alcalá de Henares 1508-Madrid 1874), Madrid, 1990, especialmente, 231-262.

4. B. G. Smith, "Gender and the Practices of Scientific History: The Seminar and Archival Research in the Nineteenth Century", The American Historical Review, 100, 1995, 1153 y 1161-1165.

5. I. Porciani, "Janus-faced Clio. Gender in the Historical Profession in Europe", en VVAA, Paths to Gender. European Historical Perspectives on Women and men, Pisa, 2010, 13; S. Berger, "Professional and Popular Historians, 1800-1900-2000”, en B. Korte y S. Paletschek (eds.), Popular History. Now and Then. International perspectives, Bielefeld, 2012, 19-21. En los países anglosajones las historiadoras tuvieron espacios dentro de la academia. Por ejemplo, la historia económica o social, entonces ámbitos poco valorados, fueron campos en los que las mujeres tuvieron peso durante el primer tercio del siglo XX: M. Berg, "The First Women Economic Historians", The Economic History Review, 45, 1992, 326. Aunque en España también hubo historiadoras semiprofesionales que exploraron estos campos de investigación, como fue el caso de María Concepción Alfaya López, quien, formada en el Centro de Estudios Históricos, recibió el apoyo del propio Rafael Altamira para que pudiese investigar sobre la cuestión social en la España del siglo XIX: M. C. Alfaya López, Noticias para la Historia económica y social de España. Teorías económico-sociales (1800 a 1820), Segovia, 1924. En el Anuario de Historia del Derecho Español de 1925 (551-553), el prestigioso historiador Eduardo Ibarra y Rodríguez aseguró que esta obra, de la entonces profesora de historia en la Escuela Normal de Segovia, era el «primer ensayo de apreciación conjunta de los influjos doctrinales en nuestro desarrollo económico» y que «cuantos, en lo futuro, hayan de tratar de estos asuntos a ella han de 
namarca y Finlandia ocho mujeres habían defendido con éxito su Tesis Doctoral en historia a la altura de 1909, y la historiadora sueca Ellen Fries fue la primera mujer de su país en conseguir un doctorado $(1883)^{6}$. Asimismo, en los Países Bajos, Johanna Naber, quien se dedicó a escribir sobre historia de las mujeres, consiguió doctorarse en $1904^{7}$.

Un caso excepcional fue el de Irlanda, ya que el primer profesor de historia moderna irlandesa en la University College Dublin fue Mary Hayden (1911), mientras que Mary Donovan O' Sullivan obtuvo tres años después un puesto en la University College Galway ${ }^{8}$. Esto fue posible porque, antes de la Gran Guerra, las historiadoras irlandesas aprovecharon que en la academia del país era una disciplina que no tenía un alto estatus. Sin embargo, cuando estas mujeres obtuvieron notoriedad, poco a poco pasaron a ser marginadas por sus pares varones ${ }^{9}$. Una situación más compleja desde el punto de vista de la categorización se vivió en el caso de aquellos lugares, como Inglaterra, en el que incluso muchos historiadores reconocidos no vivían de ello en este periodo, ya que era más bien un pasatiempo para las clases altas autofinanciadas, sobre todo hombres, pero también mujeres ${ }^{10}$. De hecho, Billie Melman ha identificado a 66 historiadoras en Inglaterra que escribieron más de 700 libros entre el siglo XIX y las primeras décadas del $\mathrm{XX}^{11}$.

En el caso español, fue desde 1910 cuando las mujeres pudieron acceder a la universidad en igualdad de condiciones formales que los hombres. Asimismo, como también se otorgó validez profesional a su título universitario de cara a los empleos que dependían del Ministerio de Instrucción Pública y Bellas Artes, desde entonces pudieron acceder al Cuerpo Facultativo de Archiveros, Bibliotecarios y Arqueólogos (CFABA), que fue uno de los organismos que más peso tuvieron en la creación de un método histórico científico centrado en las fuentes documentales archivísticas ${ }^{12}$. Sin embargo, este cambio legislativo

acudir en busca de datos e información doctrinal: mejora y sobrepuja a cuanto estaba escrito sobre el mismo argumento». Sobre esta mujer: J. M. López, Las ciencias sociales en la edad de plata española: el Centro de Estudios Históricos, 1910-1936, Madrid, Tesis Doctoral, 430.

6. M. Kaarninen, "Loneliness. Being a Woman in the Nordic Community of Historians", en P. Haapala, M. Jalava y S. Larsson (eds.), Making Nordic Historiography: Connections, Tensions and Methodology, 18501970, Nueva York-Oxford, 2017, 256-287.

7. A. Van Drenth, "Holy Beliefs and Caring Power: Josephine Butler's Influence on Abolitionism and the Women's Movement in Netherlands", en J. Daggers y D. Neal (eds.), Sex, Gender, and Religion: Josephine Butler Revisited, Nueva York, 2006, 73-96.

8. J. Harford, "The Historiography of the Professoriate: Reflections on the Role and Legacy of Professor Mary Hayden (1862-1942)", Paedagogica Historica, 55, 2019.

9. I.Porciani y M. O' Dowd, "History Women: Introduction", Rivista internazionale di storia della storiografia, 46, 2004, 3-34.

10. D. Looser, British Women Writers and the Writing of History, 1670-1820, Maryland, 2005; D. Looser, "Catharine Macaulay: The 'Female Historian' in Context", Études Épistémè, 17, 2010, 5.

11. B. Melman, "Gender, History and Memory: The Invention of Women's Past in the Nineteenth and Early Twentieth Centuries", History and Memory, 5, 1993, 9.

12. Gaceta de Madrid, 9-3-1910, 497-498; Gaceta de Madrid, 4-9-1910, 731-732. Para un análisis de los pasos y evolución de la "invención" del modelo de historiador: I. Peiró, "Los académicos de la historia o la 
de 1910 no eliminó el resto de los obstáculos a los que tenían que enfrentarse las universitarias e investigadoras españolas en relación con sus pares varones. Si a esto se le suma que el acceso a la formación académica reglada solo era posible para una minoría de la población privilegiada económicamente, el resultado es que en el curso 1927-1928 solo había 1681 universitarias en España ${ }^{13}$ y, todavía a la altura de 1954, solo 155 mujeres se habían doctorado en Filosofía y Letras ${ }^{14}$.

Si el acceso a la universidad fue muy limitado, más lo fue a la JAE, la institución más importante para el desarrollo del personal investigador en España, donde las mujeres solo obtuvieron 11 pensiones o equiparaciones a pensión para investigar sobre el pasado ${ }^{15}$, o al Centro de Estudios Históricos ( $\mathrm{CEH})$, que fue el principal centro de la investigación histórica y filosófica antes de la guerra civil ${ }^{16}$. La escasa presencia de las mujeres en estas instituciones, al igual que en la universidad, fue clave para su desplazamiento a los márgenes de la investigación histórica porque fue en estos espacios donde se desarrolló la profesionalización de los historiadores, debido a que les dio un trabajo en unas condiciones que en otros lugares no habrían encontrado. Por ejemplo, el CEH sirvió para que surgiese una nueva clase de investigadores profesionales de los estudios históricos que, alejados de corporaciones más tradicionales como la Real Academia de la Historia, progresivamente fueron copando puestos relevantes en la Universidad, en la segunda enseñanza y en el CFABA ${ }^{17}$.

El acceso tardío a las licenciaturas y a los programas de doctorado provocó que, necesariamente, las trayectorias investigadoras de las españolas fueran muy diferentes a las de los varones, por lo que en el estudio de la profesionalización de las historiadoras hay que atender a esta cuestión ${ }^{18}$. En concreto, hay que tener presente que las mujeres que escribieron sobre el pasado durante este periodo frecuentemente no se formaron en los canales oficiales de la academia. Asimismo, quienes sí lo hicieron, como era prácticamente imposible que pudiesen dedicarse profesionalmente y de forma estable a la investigación o a la docencia universita-

imagen del ideal del historiador decimonónico", Studium, 4, 1992, 83-104. Sobre la importancia del CFABA en el proceso de profesionalización de la historiografía: G. Pasamar e I. Peiró, La Escuela Superior de Diplomática. Los archiveros en la historiografía española contemporánea, Madrid, 1996.

13. C. Rodríguez López, "Experiencias universitarias en torno a 1910. En el centenario del acceso de la mujer a los estudios universitarios", CEE Participación Educativa, 15, 2010, 209-219. Un problema que no fue particular del caso español: C. Flecha, "Barreras ante las pioneras universitarias: una mirada transnacional”, CIAN. Revista de historia de las universidades, 1, 2019, 19-59.

14. M. J. Matilla, "Las primeras doctoras universitarias en España (1882-1954). La Facultad de Filosofía y Letras”, en J. Cuesta, M. L. de Prado y F. J. Rodríguez (dirs.), ¿Mujeres sabias? Mujeres universitarias en España y América Latina, Limoges, 2015, 110-119.

15. Pueden consultarse en: http://archivojae.edaddeplata.org/jae_app/. La escasa presencia/importancia de las mujeres en las secciones de historia de la JAE puede apreciarse por su omisión en: J. Gómez Mendoza (dir.), La Junta para Ampliación de Estudios e Investigaciones Científicas y los académicos de la historia, Madrid, 2008.

16. J. M. López, Heterodoxos españoles. El Centro de Estudios Históricos, 1910-1936, Madrid, 2006.

17. López, Heterodoxos... op. cit.

18. Matilla, “Las primeras doctoras..., op. cit., 110-111 y 115-119. 
ria, optaron por buscar trabajo en ámbitos que ofrecían mayores posibilidades de estabilidad profesional y económica, como el profesorado de segunda enseñanza o el CFABA ${ }^{19}$. Por este motivo, las españolas que quisieron investigar sobre el pasado frecuentemente trabajaron en los márgenes de la disciplina, tanto metodológica como temáticamente. Esta situación era conveniente para muchas porque sentían mayor reconocimiento fuera de la academia y les ofrecía la libertad necesaria para tratar aquellos temas que les interesaban ${ }^{20}$.

La situación que experimentaron las españolas no fue muy diferente a la de otros lugares. Por ejemplo, en Estados Unidos, donde el número de mujeres que se formaron en las universidades para ser historiadoras fue mayor, igualmente tuvieron un escaso peso en la academia y normalmente acabaron empleadas en institutos, bibliotecas, archivos y universidades femeninas, ya que en estos ámbitos obtenían un sustento económico más estable. No obstante, algunas mujeres consiguieron hacerse un hueco en la academia y sirvieron como referentes para las siguientes generaciones de historiadoras ${ }^{21}$.

Un caso diferente fue el de España, donde las historiadoras no tuvieron referentes sólidos en el periodo, aunque las mujeres llevaban muchos siglos escribiendo sobre el pasado $^{22}$. La primera generación de historiadoras españolas profesionales surgió durante las décadas de 1920 y 1930, que fue cuando comenzaron a licenciarse las primeras mujeres que buscaron dedicarse, con más o menos éxito, a la investigación histórica como

19. La carrera académica profesional durante el primer tercio del siglo XX estaba diseñada de una manera en la que los catedráticos controlaban la disciplina y el sistema de cooptación y de contratación de profesorado. Entonces, como los nombramientos no estaban regidos necesariamente por criterios impersonales, el sexo del candidato influía a la hora de obtener un puesto. Para la evolución durante el siglo XX de la situación de los historiadores y de la profesión de historiador: I. Peiró, Historiadores en España. Historia de la Historia y memoria de la profesión, Zaragoza, 2013, 19-84; M. Nash, "Mujeres, género y las fronteras abiertas de la historia: una cartografía intelectual”, en J. Aurell (ed.), La historia de España en primera persona. Autobiografías de historiadores hispanistas, Barcelona, 2012, 187-211; A. Burguière y B. Vincent (dirs.), Un siécle d' historiennes, París, 2014. Para obtener información sobre casos concretos: C. Flecha, "Profesoras en la Universidad. El tránsito de las pioneras en España”, Arenal, 17, 2010, 255-297; C. Flecha, "Profesoras de Historia Medieval: mirando a los orígenes”, en P. Díaz, G. Franco y M. J. Fuente (eds.), Impulsando la historia desde la historia de las mujeres: la estela de Cristina Segura, Huelva, 105-116.

20. G. Pomata, "Amateurs by Choice: Women and the Pursuit of Independent Scholarship in 20th Century Historical Writing”, Centaurus, 55, 2013, 196-210.

21. Algunos ejemplos concretos de mujeres han sido magníficamente estudiados: K. K. Sklar, "American Female Historians in Context, 1770-1930", Feminist Studies, 3, 1975, 179-181; J. Goggin, "Challenging Sexual Discrimination in the Historical Profession: Women Historians and the American Historical Association, 1890-1940", The American Historical Review, 97, 1992, 769-771 y 784. Para el estudio de las pioneras de la investigación histórica, fuesen estas profesionales o amateurs, en varios países: S. Cosner y J. R. Scanlon, American Women Historians, 1700s-1990s: A Biographical Dictionary, Westport, 1996; N. Smith, A 'Manly Study'?: Irish Women Historians 1868-1949, Nueva York, 2006; P. G. Dagbovie, "Black Women Historians from the Late 19th Century to the Dawning of the Civil Rights Movement", The Journal of African American History, 89, 2004, 242.

22. T. Robles, Historiadoras negadas: escritura femenina de historia en el largo siglo XVIII, Madrid, Tesis doctoral, 2017.

Revista de historiografía 35, 2021, pp. 171-193 
medio para ganarse la vida. No obstante, su número fue escaso y su repercusión académica reducida ${ }^{23}$. En el extenso Diccionario Akal de historiadores españoles contemporáneos (1840-1980), solo aparecen cinco mujeres historiadoras, de las cuales únicamente la medievalista Mercedes Gaibrois y Riaño (1891-1960) comenzó su trayectoria como investigadora antes de la guerra civil ${ }^{24}$. Esta mujer fue la historiadora profesional más importante y reconocida del periodo; de hecho, fue la primera que logró entrar en la Real Academia (1932) ${ }^{25}$. Asimismo, hubo algunas españolas destacadas que investigaron sobre el pasado aprovechando sus trabajos en los archivos, como fue el caso de Ángela García Rives, la primera mujer que ingresó en el CFABA, que escribió varias obras que se pueden considerar como trabajos de historiadora profesional ${ }^{26}$.

De todas formas, si bien el número de historiadoras profesionales antes de la Segunda República española fue muy reducido, como ya he señalado, hubo un grupo de mujeres cultas y de posición acomodada que, por su afición a la historia, investigaron sobre el pasado sin haber recibido una formación académica reglada para ello. Asimismo, algunas mujeres que sí estudiaron en la universidad, pero no pudieron acceder a puestos académicos en las instituciones dedicadas a la investigación histórica, escribieron sobre el pasado en los márgenes de la historiografía profesional. De nuevo, esta situación fue similar a la que experimentaron las mujeres de los otros países europeos y americanos ${ }^{27}$. En las próximas páginas, por un lado, analizaré las estrategias que desplegaron las mujeres para poder investigar sobre el pasado y publicar sus resultados. Por otro lado, prestaré atención a algunas católicas y conservadoras españolas y sus trabajos históricos más destacados, centrándome especialmente a los temas escogidos, sus intereses concretos y sus objetivos. Unos aspectos que normalmente tuvieron relación con el protagonismo de las mujeres en lo que ellas entendieron como las partes más gloriosas de la historia de España. El poder analizar esto es relevante porque, normalmente, sus obras tuvieron más impacto y difusión que los trabajos de las historiadoras profesionales.

23. A. Camino, "Historiadoras: una prosopografía de cuatro de las pioneras de la investigación histórica en España”, Hispania Nova, 16, 2018, 197-226.

24. Pasamar y Peiró, Diccionario... op. cit., 267, 280, 305, 359 y 468. Sobre Gaibrois: C. Morales García y B. Rivero, "Mercedes Gaibrois: una historiadora colombiana en el fondo Ballesteros”, en VVAA, XXI Coloquio de Historia Canario-Americana, 2016.

25. La Voz, 27-12-1932, 3; Diario de Córdoba de comercio, industria, administración, noticias y avisos, 10-3-1935, 1; El Progreso, 7-3-1935, 6. Entre sus trabajos, destaca, por ejemplo: M. Gaibrois, Historia del reinado de Sancho IV de Castilla, Madrid, 1922.

26. Á. García Rives, Fernando VI y Doña Bárbara de Braganza (1748-1759). Apuntes sobre su reinado, Madrid, Tesis Doctoral, 1917; Á. García Rives, Clases sociales en León y Castilla, Madrid, 1921.

27. I. Porciani y R. Lutz (eds.), Atlas of European Historiography: The Making of a Profession, 1800-2005, Basingstoke, 2010; H. L. Smith, M. S. Zook (eds.), Generations of Women Historians: Within and Beyond the Academy, Londres, 2018. 


\section{La vía de copar los temas y los enfoques estigmatizados por la historiografía profesional española}

Conforme se aproximó el fin del siglo XIX, se fue haciendo cada vez más nítida la delimitación entre lo que debía ser la historia científica (preocupada sobre todo por la construcción nacionalista de la historia) y la erudición localista, un proceso en el que esta última quedó desplazada a la periferia del espacio académico profesional al dejar de ser valorada. Esta erudición localista, que estaba centrada principalmente en heráldica, genealogías y biografías, aspectos sobre los que era más fácil encontrar fuentes, se quedó a cargo, ante todo, de historiadores/as semiprofesionales y amateurs ${ }^{28}$. Sin embargo, muchas de las historiadoras de este perfil que escribieron biografías y genealogías intentaron acercar su forma de investigar a la nueva metodología científica con pretensión de objetividad, por lo que también se preocuparon por la construcción nacionalista y religiosa de la historia ${ }^{29}$.

28. Ver: J. M. Sánchez Prieto, El imaginario vasco. Representaciones de una conciencia histórica, nacional y política en el escenario europeo 1833-1876, Donostia, 1994; J. A. Vallejo, Los montañeses en la Real Academia de la Historia. (1856-1936). Una aportación metodológica a la historia de la historiografía regional de Cantabria, Santander 1993; J. Fontana, "La historiografía española del siglo XIX. Un siglo de renovación entre dos rupturas”, en S. Castillo (coord.), La historia social en España. Actualidad y perspectivas, Madrid, 1991; Pasamar y Peiró, Diccionario... op. cit., 35-36. Por poner varios ejemplos concretos de una autora, Emilia Pardo Bazán escribió biografías tanto de hombres como de mujeres destacadas como Madame du Barry, Juana la Loca o la Venerable de Agreda: E. Pardo Bazán, Hombres y Mujeres de Antaño, Barcelona, s/f; E. Pardo Bazán, El P. Luis Coloma: biografía y estudio crítico, Madrid, 1891; E. Pardo Bazán, San Francisco de Asís (siglo XIII), Madrid, 1903. Asimismo, Pardo Bazán ayudó a reeditar y prologó un libro de María de Jesús de Ágreda: Vida de la Virgen, Barcelona, 1899. Ver también: L. Cuesta Gutiérrez, La obra de D. Pedro de la Gasca en América. Contribución al estudio de la política colonizadora de España en América durante el siglo XVI, Santiago, 1928.

29. Algunos ejemplos de esta tendencia fueron, en primer lugar, la carlista Dolores de Gortázar: D. de Gortázar, "San Miguel de Escalada. Nuevas ilustraciones de su historia moderna", Boletín de la Real Academia de la Historia, 34, 1899, 266-268. En segundo lugar, la propagandista católica Eulàlia Anzizu, quien escribió varios estudios históricos que buscaban poner en valor diversas arquitecturas religiosas: E. Anzizu, Fulles historiques del Real Monestir de Santa María de Pedralbes, Barcelona, 1897. Sobre su figura se ha escrito bastante en los últimos años: M. C. Bernal, Eulària Anzizu: poeta, arxivera i historiadora, Vic, 2013 ; C. Aixalà, M. C. Bernal y A. Castellano-Tresserra, Dones silenciades: el llegat de sor Eulària Anzizu (1868-1916) al monestir de Pedralbes, Barcelona, 2018. En tercer lugar, la archivera H. Lo Cascio-Loureiro, Historia de Madrid (Episodios 1561-1932) e Historia documentada de la antigua Iglesia hospital de S. Pedro y S. Pablo, de Madrid, Madrid, 1932. En cuarto lugar, las obras de Concha Muedra: C. Muedra Benedito, Adiciones al Fuero de Medina del Campo, Madrid, 1929; C. Muedra Benedito, Nuevas Behetrías de León y Galicia y textos para el estudio de la Curia regia leonesa, Madrid, 1930. Por último, la maestra católica sevillana Amantina Cobos, quien escribió el libro Sevilla en el siglo XVI, del que no se conservan ejemplares, en el cual abordó un estudio histórico sobre las cofradías y procesiones en esa ciudad: Diario de la Marina, 15-8-1926, 40; Diario de Córdoba de comercio, industria, administración, noticias y avisos, 2-4-1926, 2. Asimismo, escribió muchas historias sobre las grandes arquitecturas de Andalucía, como Medina Azahara y la Mezquita de Córdoba, pero también de otras regiones, con el objetivo de exaltarlas con un espíritu patriótico: Diario 
Las mujeres católicas y conservadoras aprovecharon esta estrategia para justificar mediante su contribución a la nación (a cuyo engrandecimiento y exaltación colaboraban con sus textos históricos) una serie de derechos en tanto que mujeres. Por tanto, intentaron colaborar en el proceso de la construcción de la nación española a la vez que trataron de definir su identidad como mujeres en relación con la propia nación. Sus antepasadas españolas, con las que establecían una relación de descendencia directa, habían escrito, a su juicio, algunas de las páginas más importantes de la historia «nacional» en defensa de la religión y del espíritu patriótico, lo cual debía servir de ejemplo a las mujeres del presente. Entonces, al igual que el vínculo entre la feminidad y el catolicismo había animado a las mujeres piadosas para implicarse en el proyecto de regeneración social católica, la historia, como un elemento central en el proyecto de construcción nacional de las derechas católicas, estimuló que las historiadoras católicas semiprofesionales y aficionadas sintiesen una necesidad por resaltar el valor y el espíritu de sacrificio de las mujeres en los momentos más importantes y decisivos de la historia de España ${ }^{30}$.

Las historiadoras católicas semiprofesionales y aficionadas tuvieron una agenda predeterminada a la hora de abordar sus investigaciones sobre el pasado. Por este motivo, la selección de sus temas, el desarrollo de sus ensayos y sus conclusiones no pueden entenderse sin tener en cuenta que en las autoras predominaba una identidad católica, nacional y de mujeres, aunque estas no siempre estuviesen jerarquizadas en el mismo orden. Estas identidades impregnaban el conjunto de sus trabajos históricos en todo momento, pues marcaban sus preguntas y objetivos, mientras que determinaban buena parte de las respuestas que ofrecían a sus lectores.

En cualquier caso, los análisis de las historiadoras aficionadas y semiprofesionales durante el primer tercio del siglo XX no se diferenciaron mucho de los trabajos de los historiadores profesionales. La propia Mercedes Gaibrois, quien era defensora del positivismo histórico, hizo en sus trabajos históricos afirmaciones como que el mundo fue creado por Dios, quien cuidaba especialmente a España, o que Benito Mussolini fundó el partido fascista en Italia para defender la civilización cristiana. En ambos casos unas ideas muy similares a las defendidas por la propagandista política Carmen Velacoracho en sus biografías sobre Hitler y Mussolini ${ }^{31}$. Esto, sin embargo, no implica que Gaibrois no fuese una historiadora seria y

de Córdoba de comercio, industria, administración, noticias y avisos, 30-4-1917, 1; Diario de Córdoba de comercio, industria, administración, noticias y avisos, 6-7-1916, 6; Diario de Burgos, 24-12-1918, 1. Esta fue una tendencia que también se dio en el plano transnacional: K. K. Sklar, "American Female Historians...", op. cit., 177; B. G. Smith, "The Contribution of Women to Modern Historiography in Great Britain, France, and the United States, 1750-1940", The American Historical Review, 89, 1984, 720.

30. N. Aresti, "A Fight for Real Men: Gender and Nation-Building during the Primo de Rivera Dictatorship (1923-1930)", European History Quarterly, 50, 2020, 255 y 262-263. Esto también ocurrió a nivel transnacional: Porciani, "Janus-faced Clio..., op. cit., 12-13.

31. M. Gaibrois, Breve Historia de España, Madrid, 1940, 7-8, 57 y 98; G. Pasamar, Apologia and Criticism: Historians and the History of Spain, 1500-2000, Bern, 2010, 192. Sobre las biografías escritas por Carmen Velacoracho: A. Camino, "Carmen Velacoracho: una pro-nazi católica en el primer franquismo 
rigurosa, ya que sus planteamientos se enmarcaban en las corrientes de la historiografía académica española del momento.

Los principales académicos del periodo buscaron la mayor objetividad posible en el análisis de las fuentes mediante las técnicas que les ofrecía el positivismo metodológico, consistente en la búsqueda de documentos, su transcripción, su crítica y su confrontación con otros $^{32}$. Sin embargo, todos tuvieron una agenda específica detrás de sus investigaciones. Por ejemplo, Marcelino Menéndez Pelayo, el positivista que se convirtió en gran referente de la historiografía conservadora española, buscó en el pasado las raíces sobre las que se asentaba la supuesta consustancialidad de la nación española, señalando al catolicismo como elemento vertebrador de la misma, por lo que interrelacionaba la historia del país con la de la Iglesia católica $^{33}$. Mientras, el historiador Cánovas del Castillo, quien fue presidente del Consejo de Ministros en varios periodos, consideraba que las naciones tenían una misión confiada a lo largo de la historia por Dios o por la naturaleza ${ }^{34}$. Por último, otros autores como Rafael Altamira, Antonio Ballesteros (marido de Mercedes Gaibrois) o Pedro Aguado, consideraron que sus escritos debían promover la defensa de la historia española y su cultura e identidad ${ }^{35}$. Estas ideas sobre el uso instrumental de la historia influyeron en los trabajos de las historiadoras católicas y conservadoras semiprofesionales y aficionadas, que entendían que la defensa del catolicismo y de la patria eran los valores centrales de su cultura política.

Los trabajos de estas historiadoras, aparte de estar centrados en estudios locales y en genealogías y biografías, también aprovecharon otro espacio que la historiografía profesional española del periodo había dejado relegado: el análisis de la contemporaneidad. Para los académicos españoles, fueron los siglos XV, XVI y XVII aquellos en los que se debía focalizar la atención, ya que permitían estudiar los secretos de la «grandeza» $\mathrm{y}$ «decadencia» de los españoles en dichas centurias. En contraposición, el siglo XIX fue generalmente pasado por alto, salvo episodios concretos de la guerra de la independencia o de las guerras carlistas ${ }^{36}$. Este

(1939-1944)", en A. Fiuza y G. Grecco (orgs.), Escrituras de autoria feminina e identidades ibero-americanas, Rio de Janeiro-Recife-Madrid, 2020, 119-144.

32. Para el proceso de profesionalización: Pasamar y Peiró, Diccionario... op. cit., 12-13 y 30-38.

33. A. Santoveña Setién, Marcelino Menéndez Pelayo: revisión crítico-biográfica de un pensador católico, Santander, 1994, 86-89 y 235; L. Marco Sola, "El catolicismo identitario en la construcción de la idea de nación española. Menéndez Pelayo y su 'Historia de los Heterodoxos Españoles'”, Ilu, 14, 2009, 101-116. También hubo muchas narrativas históricas que vincularon estrechamente la historia de España con la monarquía.

34. Pasamar, Apologia and... op. cit., 7 y 94-99.

35. Pasamar, Apologia and... op. cit., 142 y 162-165.

36. J. M. Cuenca Toribio, “La historiografía española sobre la Edad Contemporánea”, en José AndrésGallego (coord.), Historia de la historiografía española: Nueva edición revisada y aumentada, Madrid, 188; J. M. Jover Zamora, Historiadores españoles de nuestro siglo, Madrid, 1999, 29. No obstante, la denostación del pasado inmediato no existió con anterioridad a la profesionalización del trabajo de historiador: Jover, Historiadores... op. cit., 35-36. Para algunos trabajos contemporáneos importantes sobre la historia inmediata: Conde de Toreno, Historia del levantamiento, guerra y revolución de España, Tomo I, Madrid, 1835 o J. de Burgos, Anales del Reinado de Da Isabel II, Madrid, 1850-1851. 
desprestigio y desatención fue aprovechado por algunas historiadoras aficionadas o semiprofesionales para explorar el pasado cercano con libertad, tal y como hicieron, por ejemplo, sus pares italianas ${ }^{37}$. Algunas mujeres, como la periodista y escritora católica Sofía Casanova, incluso aprovecharon su reconocimiento público para escribir sobre un pasado reciente que, en ocasiones, vivieron en primera persona. Casanova escribió En la corte de los zares (1929), un relato histórico que abarcaba desde los zares antiguos hasta el inicio de la revolución rusa, un acontecimiento que se produjo cuando ella se encontraba como periodista en el frente ruso cubriendo la Gran Guerra ${ }^{38}$.

Estas estrategias, si bien fueron la consecuencia de que las mujeres estuviesen relegadas a los márgenes de la historiografía profesional, provocaron que las historiadoras en pocas ocasiones tuviesen la oportunidad de publicar sus trabajos en las revistas exponentes de la erudición histórica de la Restauración, como La Revista de Archivos, Bibliotecas y Museos, la Revista Histórica o el Boletín de la Real Academia de la Historia. Una de las mujeres que más publicó en estas revistas fue la carlista Dolores de Gortázar, quien escribió durante su juventud algunos artículos que fueron frecuentemente citados, lo que demuestra que sus pares historiadores los consideraron como trabajos válidos ${ }^{39}$. El primero de sus escritos fue un estudio descriptivo del culto y el sepulcro de San Miguel de Escalada, en el que presentó la lista de priores de aquel lugar entre los siglos XVIII y XIX ${ }^{40}$. El segundo artículo lo publicó para ampliar y corregir algunos de los

37. Ilaria Porciani, "Women and the Writing of National History. Italy, 1860-1914", en VVAA, Political Systems and Definitions of Gender Roles, Pisa, 2001, 109. Sobre alguna de estas españolas: A. M. Velasco Molpeceres, "Enseñanza en inclusión: las madres de la historiografía americana en España. La obra de Fanny Calderón de la Barca, Faustina Sáez de Melgar, la Baronesa de Wilson y Eva Canel”, J. Gómez, E. López, L. Molina García (coords.), Research foundations of the social sciences, Puerto Rico, 2016, 102-107. Para varios ejemplos específicos: E. Serrano, América y sus mujeres. Estudios hechos sobre el terreno, Barcelona, 1890; E. Serrano, Americanos célebres. Glorias del Nuevo Mundo, Barcelona, 1888; F. Sáez de Melgar (dir.), Las mujeres españolas, americanas y lusitanas pintadas por sí mismas, Barcelona, 1881; A. García Llansó, R. Pomés, E. Serrano y A. Opisso, Historia de la mujer contemporánea, Barcelona, 1899; S. Acosta de Samper, La mujer en la sociedad moderna, París, 1895; F. Pérez Cerpa, Salteras en la Guerra de la Independencia (recuerdo en el primer Centenario), Sevilla, 1908.

38. S. Casanova, En la corte de los Zares, Madrid, 1929 [1925]. Para algunas reseñas de la obra en las que la autora fue alabada: El Eco de Santiago, 26-7-1919, 2; La Libertad, 18-3-1925, 4. Para una visión más personal sobre los acontecimientos que vivió: S. Casanova, La revolución bolchevista: diario de un testigo, Madrid, 1920.

39. F. Fita, "Tres aras antiguas de San Miguel de Escalada”, Boletín de la Real Academia de Historia, 33, 1898, 221; M. Gómez-Moreno, Catálogo monumental de España Provincia de León (1906-1908), Madrid, 1925-1926, 112-114; F. Abad León, “Expansión de la vida eremítica y monástica en La Rioja”, Antigüedad y cristianismo, 16, 1999, 293; F. Cantera Burgos, Fuero de Miranda de Ebro, Madrid, 1945, 92; A. Calvo, El monasterio de Gradefes. Apuntes para su historia y la de algunos otros cenobios y pueblos del Concejo, León, 1936-1944, 47; J. Cantera Orive, "La ermita de San Pedro de Torrecilla de Cameros”, Berceo, 45, 1957, 438.

40. D. de Gortázar, "San Miguel de Escalada. Nuevos monumentos y documentos”, Boletín de la Real Academia de la Historia, 33, 1898, 225-234. 
datos que había ofrecido en el trabajo anterior a la luz de nuevos documentos consultados ${ }^{41}$. Por último, en otro de sus trabajos se centró en repasar de forma pormenorizada los fondos del archivo del monasterio de Valvanera y, además, explicitó que para ella los trabajos históricos debían aspirar a sembrar «el puro grano de la verdad $»^{42}$.

\section{Historiadoras de las mujeres}

Los historiadores varones fueron durante el siglo XIX y el primer tercio del siglo XX los guardianes de una profesión que poco a poco se fue dotando de una aureola de cientificidad. Entre otras cosas, definieron que la disciplina académica debía centrarse fundamentalmente en una serie de aspectos, principalmente políticos, que, generalmente, habían sido copados por hombres. Por tanto, no solo fueron prácticamente excluidas las mujeres de la profesión, sino que también se consideró que, aquellos espacios en los que habían tenido en el pasado mayor protagonismo, no eran merecedores de recibir una atención pormenorizada por parte de la disciplina académica profesional, por lo que el estudio de estas cuestiones quedó restringido a los círculos historiográficos semiprofesionales y aficionados ${ }^{43}$.

Sin embargo, las mujeres habían tenido presencia en los estudios sobre el pasado antes de que se iniciase el proceso de profesionalización de la historiografía, por lo que muchas historiadoras semiprofesionales y aficionadas las siguieron incluyendo en sus trabajos durante el primer tercio del siglo XX ${ }^{44}$. Por este motivo, Gianna Pomata contradijo en 1990 la asumida idea, surgida de las historiadoras feministas de las décadas de 1970 y 1980, sobre que las mujeres habían sido siempre invisibilizadas por la historiografía occidental. Lo que defendió la historiadora italiana fue que, si bien era una afirmación cierta en lo referente a la disciplina histórica profesional y académica reglada, no lo era fuera de estos ámbitos. En una corriente historiográfica cuyas autoras fueron fundamentalmente mujeres, estos trabajos contribuyeron a la construcción histórica del pasado a la vez que incluyeron a las mujeres en los relatos como parte de colectivos (la familia, la nación o la comunidad religiosa) o como sujetos garantes de atención histórica específica, especialmente mediante la fórmula de narrar las vidas

41. D. de Gortázar, "San Miguel de Escalada. Nuevas ilustraciones de su historia moderna", Boletín de la Real Academia de la Historia, 34, 1899, 266-268. La tendencia a "rescatar" documentos históricos también estuvo extendida entre otras historiadoras españolas, independientemente de que fuesen de un corte más profesionalizado o aficionado: M. I. de Lersundi y Blanco, Algunos documentos curiosos para la Historia de España y una instancia, San Sebastián, 1929.

42. D. de Gortázar, "El monasterio de Valvanera. Índices de su Becerro y Archivo a mediados del siglo XVII", Boletín de la Real Academia de la Historia, 51, 1907, 241-306.

43. J. Goggin, “Challenging Sexual Discrimination..., op. cit., 778-781; A. Epple, “Questioning the Canon: Popular Historiography by Women in Britain and Germany (1750-1850)", en S. Paletschek (ed.), New German Historical Perspectives, Oxford/New York, 2011, 32.

44. K. K. Sklar, “American Female Historians..., op. cit., 176-177; B. Smith, Men, Women and Historical Practice, Harvard, 1998. 
de mujeres célebres ${ }^{45}$. Aunque también fue habitual que, cuando se hacían narraciones sobre el pasado de una nación, los historiadores varones mencionasen a las mujeres célebres y excepcionales como signo de que su país era tan avanzado que hasta sus mujeres hacían cosas relevantes. Incluso, en ocasiones, publicaron trabajos monográficos, sobre todo en forma de biografías, que tenían a las mujeres como únicas protagonistas ${ }^{46}$.

Esta tendencia estuvo muy extendida y, de hecho, en las primeras décadas del siglo XX fue habitual que todas las historiadoras escribiesen algunos trabajos relacionados con mujeres $^{47}$. Incluso, a veces se atrevieron a criticar a los hombres que no las incluían en sus trabajos, históricos o literarios. Por ejemplo, Concepción Saiz Otero criticó a Benito Pérez Galdós por la exclusión de las mujeres en sus Episodios nacionales:

Mas tal vez porque las mujeres honradas no tienen historia (aunque contribuyen a hacer la de los hombres), el insigne autor de los Episodios descuidó estudiar, en alguno de los dedicados a la época revolucionaria, la influencia decisiva ejercida por la Revolución de 1868 sobre la cultura de la mujer y la transformación social ocasionada por esta influencia ${ }^{48}$.

Los estudios de historia de las mujeres en España, como en otras historiografías, pronto se aliaron con el enfoque biográfico ${ }^{49}$. Esto se debió a que, por un lado, pervivía el interés

45. G. Pomata, "Storia particolare e storia generale. In margine ad alcuni manuali di storia delle donne”, Quaderni Storici, 74, 1990, 341-385. Ver también: M. Bolufer, Mujeres y hombres en la Historia. Una propuesta historiográfica y docente, Granada, 2018, 1-39; M. Bolufer, "Multitudes del yo: biografía e historia de las mujeres", Ayer, 93, 2014, 89; N. Z. Davis, "Gender and Genre. Women as Historical Writers", en P. Labalme (ed.), Beyond their Sex. Learned Women of the European Past, Nueva York, 1980, 153-182.

46. R. Pomés, A. Opisso y E. M. Vilarrasa, Historia de la mujer a través de los siglos, Barcelona, 1899; J. I. Valentí, La mujer en la historia, Palma de Mallorca, 1896; V. Díez Canseco, Diccionario biográfico universal de mujeres célebres, Madrid, 1844; M. Poza Rodríguez, Mujeres célebres aragonesas, Zaragoza, 1884; E. Castelar, Galería histórica de mujeres célebres, Madrid, 1888; J. Gómez de Arteche, La mujer en la Guerra de la Independencia, Madrid, 1903; J. Gómez de Arteche, La mujer española en el trono. Conferencia dada en el Círculo Patronato de San Luis Gonzaga el 14 de marzo de 1904, s/c, 1906. Asimismo, el historiador alemán Heinrich Finke, especialista en temas de la Iglesia católica y de la Península Ibérica, publicó una obra sobre la historia de las mujeres que el prestigioso historiador Ramón Carande tradujo al castellano: H. Finke, $L a$ mujer en la edad media, Madrid, 1926.

47. Smith, “The Contribution..., op. cit., 709-710, 726 y 730; Sklar, "American Female Historians...”, op. cit., 177; Berg, “The First Women...”, op. cit., 311-313 y 317-322; D. R. Woolf, "A Feminine Past? Gender, Genre, and Historical Knowledge in England, 1500-1800", The American Historical Review, 102, 1997, 677 679.

48. C. Saiz Otero, La Revolución del 68 y la cultura femenina. Un episodio nacional que no escribió Pérez Galdós, Madrid, 1929, 12.

49. Porciani, "Women and the Writing..., op. cit., 109; L. Berglund, “A Medieval Heroine for the Modern Woman", Scandinavian Journal of History, 42, 2017, 46-70; T. Kinnunen, "Feminist Biography in Finland and Sweden around 1900: Creation of Bonds of Admiration and Gratitude", en A. Schaser, S. Schraut y P. Steymans-Kurz (eds.), Erinnern, vergessen, umdeuten?: Europäische Frauenbewegungen im 19 und 20, Frankfurt/Nueva York, 2019, 312-337; Smith, “The Contribution...”, op. cit., 714-718. 
por rendir culto a las grandes figuras del pasado, también a las que eran mujeres. Por otro lado, resultaban útiles para que las lectoras tuviesen referentes moralizantes e inspiradores en los que fijarse, lo que reforzaba una identidad particular en tanto que mujeres, a la vez que se abordaban, de forma más o menos explícita, los obstáculos adicionales que en su vida encontraron por el hecho de ser mujeres ${ }^{50}$. Esto tiene relación con que, durante este periodo, frecuentemente los estudios históricos realizados por historiadoras semiprofesionales o aficionadas a nivel transnacional estuvieron conectados con las diferentes ramas del feminismo y sus reivindicaciones, aunque no todas las autoras fueron en su vida militantes feministas ${ }^{51}$. Las razones por las que cada historiadora optó por estudiar a una determinada mujer del pasado, así como los aspectos de su biografía en los que se centró, variaron con el paso tiempo y en función de los intereses personales de cada autora. Esto se debe a que existe un innegable presentismo en el ejercicio del oficio de historiador/a que es necesario tener en cuenta para no caer en el ahistoricismo. Sin embargo, en las próximas páginas me centraré en las características generales, ya que especificar las razones por las que cada historiadora seleccionó un tema o una mujer para su estudio del pasado y exponer en qué cuestiones específicas se focalizó, es un esfuerzo que excedería las dimensiones espaciales del presente ensayo.

Si bien el principal objetivo de estos estudios de las historiadoras semiprofesionales o amateurs era crear una conexión entre el pasado y el presente (ya que se sentían herederas directas de las mujeres destacadas), estos trabajos normalmente fueron fruto de investigaciones rigurosas que se rigieron, a rasgos generales, por los métodos y cánones demandados por la academia del periodo ${ }^{52}$. Asimismo, algunas historiadoras profesionales también abordaron estas temáticas desde dicho enfoque. Entre ellas destaca el caso de Aurea Javierre Mur, una de las pioneras del CFABA. Aunque su larga y prolífica trayectoria investigadora se concentró principalmente en la época posterior a la guerra civil, en el periodo anterior publicó una biografía sobre Marta de Armagnac, la esposa de Juan I de Aragón, que fue fruto de su tesis

50. Por citar algunas de estas obras escritas por mujeres: M. P. Sinués, Mujeres ilustres. Narraciones histórico-biográficas, Madrid, 1884 [1864]; L. C. Monreal, La educación de las niñas por la historia de españolas ilustres, Madrid, 1873; C. Gimeno de Flaquer, Mujeres: vidas paralelas, Madrid, 1893; M. L. Morales, Algunas mujeres, Gerona, 1932; E. Sánchez de Arrojo, Mujer y reina: ligero esbozo de las virtudes de Isabel la Católica, Guadalajara, 1930. También la alemana Elizabeth Busse-Wilson se doctoró en 1914 con una biografía sobre Isabel de Hungría. Sobre ella: Pomata: “Amateurs by Choice...”, op. cit., 201. Ver también: R. G. Sumillera, "Historiadoras británicas del XIX: biografía y edición en las obras de Agnes y Elizabeth Strickland y Mary Anne Everett Green”, Quaderns de Filologia: Estudis Literaris, 22, 2017, 77. Por último, también hubo mujeres no católicas y conservadoras, como la escritora conocida bajo el seudónimo Celsia Regis, que escribieron sobre las grandes figuras de referencia para el catolicismo femenino español: C. González Ramos, Isabel la Católica, Madrid, 1923.

51. Sklar, “American Female Historians..., op. cit., 171-173 y 179-181; Goggin, "Challenging Sexual Discrimination...", op. cit., 778-781; B. Dabby, "Hannah Lawrance and the Claims of Women's History in Nineteenth-Century England", The Historical Journal, 53, 2010, 699 y 703-704; Porciani, "Janus-faced Clio...", op. cit., 12-13.

52. Dagbovie, “Black Women Historians...”, op. cit., 243-253. 
doctoral $(1927)^{53}$. La elección de este tema respondió a su interés por recuperar y visibilizar a las mujeres en la historiografía ${ }^{54}$.

El recurso a las mujeres heroicas del pasado, como Isabel la Católica, Santa Teresa de Jesús o Agustina de Aragón, fue utilizado por parte de las mujeres católicas y conservadoras para justificar y legitimar con argumentos históricos la capacidad de las mujeres para intervenir en la sociedad y de movilizarse por la patria. Esta estrategia estuvo aceptada por el conjunto de la población del momento, por lo que, en un contexto de auge de la sociedad de masas, fue ampliamente utilizada como instrumento movilizador, tanto en mítines políticos como en obras literarias ${ }^{55}$. Aunque las biografías y hagiografías sobre santas fueron muy habituales en España, al igual que ocurrió en otros países, como el caso de Juana de Arco en Francia, fue Santa Teresa de Jesús quien recibió atención preferente por parte de las historiadoras semiprofesionales y aficionadas pertenecientes a las culturas políticas católicas y conservadoras, ya que ofrecía la posibilidad de transmitir a las lectoras tanto el sentimiento católico como el patriótico ${ }^{56}$.

La tendencia de escribir sobre el pasado para educar en virtudes a las lectoras del presente también se aprecia en la historiadora española profesional más importante del periodo, Mercedes Gaibrois. Esta autora frecuentemente reflejó en sus trabajos sobre mujeres españolas y latinoamericanas lo que para ella significaba ser una mujer ideal: religiosidad, caridad, patriotismo, inteligencia, labor maternal y atención al marido. A pesar de su método positivista, recomendaba a sus potenciales lectoras que se fijasen en las virtudes que señalaba de sus sujetos de estudio, a quienes identificaba como modelos: Isabel la Católica, María de Molina, María de Hungría, María la Grande, Mencia de Portugal o Sor María de Agreda ${ }^{57}$. También la

53. A. Javierre Mur, Matha de Armanyach, Duquesa de Gerona, Madrid, 1930.

54. M. P. Benítez Marco, La turolense Áurea Lucinda Javierre Mur: abriendo caminos de mujer, Teruel, 2017, 56. Ver también: M. P. Benítez Marco, “Áurea Lucinda Javierre Mur (1898-1980). Su ideal feminista católico", en A. Cenarro y R. Illion (coords.), Feminismos: contribuciones desde la historia, Zaragoza, 2014, 159-188.

55. D. de Gortázar Serantes, La roca del amor, Madrid, 1924, 39.

56. J. Estévez de García del Canto, Máximas y reglas de conducta aplicables á los diversos estados y condiciones de la vida sacadas de las obras de Santa Teresa de Jesús. Precedidas de un prólogo y un compendio de la historia de la Santa, Salamanca, 1888; I. Cheix y Martínez, Historia de Santa Teresa de Jesús, Madrid, 1893; M. de Santiago Fuentes, Santa Teresa de Jesús, Barcelona, 1918. Sobre Blanca de los Ríos, quien escribió frecuentemente sobre esta santa, por ejemplo: B. de los Ríos, Santa Teresa de Jesús y su Apostolado de Amor, Madrid, 1921; La Basílica Teresiana, 1-4-1917, 4-7; La Basílica Teresiana, 1-5-1917, 10. Elena Fortún escribió una sátira sobre la influencia que tenían estos modelos santos de conducta sobre las jóvenes lectoras: E. Fortún, Celia en el Colegio, Madrid, 1939 [1932]. Para un estudio de las hagiografías de santas realizadas por Emilia Pardo Bazán, a las que presentó ante sus lectoras como ejemplos a seguir: N. Fernández Rodríguez, "Santas de revista: Hagiografía y feminismo en Emilia Pardo Bazán”, Hispanic Research Journal, 21, 2, 2020, 101-114. Esta tendencia no fue una excepcionalidad de España: S. A. Curtis, "Writing the Lives of Saints: Archives and the Ownership of History", French Historical Studies, 40, 2, 2017, 241-266.

57. M. Gaibrois, Presencia de la mujer en la conquista de América, Madrid, 1940. Ver también: M. Gaibrois, Las jornadas de María de Hungría (1606-1646). Conferencia leída, Madrid, 1926; M. Gaibrois, La 
maestra conservadora sevillana Amantina Cobos de Villalobos, quien publicó algunas obras literarias impregnadas de temática histórica, escribió una obra titulada Mujeres célebres sevillanas (1917) en la que presentó a mujeres que debían servir como ejemplos a seguir por sus lectoras. A su juicio, si estas sevillanas fueron dechados de virtudes se debió, sobre todo, a que durante su vida siempre antepusieron su catolicismo a cualquier otra cuestión ${ }^{58}$.

La autora de la cultura política católica que más profundizó en esta dinámica fue Concepción Gimeno de Flaquer, quien durante toda su vida escribió genealogías de mujeres, del pasado o contemporáneas, que consideraba que debían ser referentes para otras mujeres. Esto lo puso en práctica tanto en sus ensayos como en El Álbum de la mujer, una revista que dirigió y en la que reprodujo frecuentemente pequeñas biografías que debían vindicar a sus protagonistas y servir de ejemplo para las lectoras. Esta estrategia fue seguida en las décadas siguientes por otras revistas dirigidas por mujeres católicas y conservadoras, como fue el caso de Mujeres Españolas.

En una fecha tan temprana como 1877, Concepción Gimeno de Flaquer publicó La mujer española, una obra en la que biografió a algunas de las mujeres más destacadas de la historia de España con el objetivo de darlas a conocer para que sirviesen como referentes a las lectoras. En estas semblanzas partió de la base de que las biografiadas demostraban que no existía una inferioridad intelectual de las mujeres en relación con los hombres cuando habían podido acceder a la educación, ya que habían hecho aportaciones políticas, sociales y culturales que habían tenido gran trascendencia ${ }^{59}$. Asimismo, presentó las contradicciones de las mujeres católicas y conservadoras del pasado a la hora de defender el acceso de las españolas a la educación y a la igualdad legal, ya que a la vez solían considerar que las mujeres tenían como papel fundamental el ejercer de esposas y madres ${ }^{60}$. Años después, Concepción Gimeno publicó Mujeres. Vidas Paralelas (1893), donde estudió biográficamente a una serie de mujeres a través de la estructura popularizada por Plutarco ${ }^{61}$. Más adelante escribió Mujeres de regia estirpe (1907), una obra en la que, a través de una serie de biografías a mujeres con

Reina Doña Mencia, Coimbra, 1930.

58. A. Cobos de Villalobos, Mujeres célebres sevillanas, Sevilla, 1917. Una obra que tuvo buena acogida en la prensa de la época: Mundo Gráfico, 20- 2-1918, 23; Andalucía, Revista ilustrada, 1-12-1925, 30-31. Sobre las obras literarias impregnadas de temática histórica: A. Cobos de Villalobos, Romances caballerescos, Sevilla, 1924. Ver también: La voz, 21-1-1925, 5; Diario de la Marina, 15-8-1926, 40; Diario de la Marina, 15-81926, 40. El escribir novelas históricas también fue habitual para las mujeres escritoras en el contexto internacional: D. Wallace, The Woman's Historical Novel: British Women Writers, 1900-2000, Nueva York, 2004.

59. C. Gimeno, La mujer española. Estudios acerca de su educación y sus facultades intelectuales, Madrid, 1877. Sobre estas cuestiones: M. Pintos, Concepción Gimeno de Flaquer. Del sí de las niñas al yo de las mujeres, Madrid, 2016, 39, 64 y 123.

60. El libro de C. Gimeno, Madres de hombres célebres, Madrid, 1895, explicita mucho más estas ideas. Esta tendencia se dio a nivel transnacional, como lo prueba el libro de Constantino Holl prologado en su traducción al español por la líder del sindicalismo católico femenino español María de Echarri: C. Holl, Mujeres ilustres. Lecturas para jóvenes católicas, Herder, 1923.

61. Este tipo de trabajos biográficos, en cuanto a su estructura, también fueron desarrollados por mujeres de otros países. Véase: Porciani, "Women and the Writing...”, op. cit., 113. 
poder político, buscaba demostrar que las mujeres en su conjunto eran aptas para la labor de gobierno, y Mujeres de raza latina (1904), en la que realizó la semblanza de una serie de mujeres de diversos países del sur de Europa y América que, según ella, se habían distinguido en sus campos de especialización. Por mucho que en la prensa se asegurase la fidelidad y rigor histórico de sus obras ${ }^{62}$, Gimeno de Flaquer buscó constantemente apoyar sus ideas feministas mediante la investigación histórica.

También hubo mujeres católicas, como Pilar Pascual de San Juan o Luciana Casilda Monreal, que pusieron especial atención en escribir libros de historia para los niños y niñas. El objetivo era que de forma amena y ligera aprendiesen la historia, patria y universal, y extrajesen lecciones morales de los grandes acontecimientos del pasado ${ }^{63}$. En el análisis que desarrollaron sobre las distintas épocas históricas abordaron la situación social de las mujeres. Por ejemplo, en Cartilla de historia de España para uso de las escuelas de niñas (1887), de Monreal, en los cuestionarios de repaso incluidos al final de cada tema se hacían preguntas como «¿Qué influencia é ilustración tenía la mujer en aquellos tiempos?», «¿De qué consideración social gozaba la mujer antes del Cristianismo?», «¿De qué consideración empezó á gozar la mujer en esta época? - ¿Hubo mujeres que influyeron poderosamente en los asuntos del Estado? - ¿Quiénes fueron éstas?», «¿Qué parte correspondió a la mujer en la civilización de los pueblos cristianos? - ¿Qué reinas y Señoras se distinguieron en ellos?» ${ }^{64}$. La respuesta a estas preguntas se encontraba más en el presente que en el pasado. Los sectores católicos y conservadores del periodo se aprovecharon del orientalismo de las corrientes anticlericales, así como de la noción ilustrada y secularizada sobre la superioridad europea, para defender que la cristiandad dignificó a las mujeres y, en consecuencia, mejoró su situación colocándolas en una posición mejor de la que tenían en el resto de los lugares. Esta idea, que fue un argumento fundamental dentro del ideario conservador y católico de la época, estuvo tan extendida a comienzos del siglo XX que los/as anticlericales de la época se vieron en la necesidad de luchar para cuestionar esa creencia. Por ejemplo, recurrieron a ejemplos del pasado medieval para intentar demostrar el menosprecio eclesiástico por las mujeres ${ }^{65}$.

Un caso diferente al resto fue el de Magdalena Santiago Fuentes, a quien como fue una profesora de historia en la Escuela Superior del Magisterio se le puede considerar una histo-

62. La correspondencia de España, 22-5-1907.

63. P. Pascual de Sanjuán, Preceptos morales para la infancia basados en hechos históricos, Barcelona, 1864; P. Pascual de Sanjuán, La moral de la historia. Colección de cuadros históricos con su aplicación moral al alcance de los niños, Barcelona, 1869; P. Pascual de Sanjuán, A través del mar: cartas sobre Historia de un padre á su hijo, Madrid, 1893; M. Orberá y Carrión, Nociones de historia de España, Valencia, 1878; Monreal, La educación de las niñas por la historia... op. cit.; L. C. Monreal, La educación de las niñas por las biografías de españolas y americanas ilustres, Barcelona, 1892; L. C. Monreal, Españolas y americanas ilustres, Madrid, 1908.

64. L. C. Monreal, Cartilla de historia de España para uso de las escuelas de niñas, Barcelona, 1887, 14, 31, 46, 91, respectivamente.

65. P. Salomón, “Laicismo, género y religión. Perspectivas historiográficas”, Ayer, 61, 2006, 307. 
riadora semiprofesional ${ }^{66}$. Desde su perspectiva, la historia se enseñaba mal en las aulas, ya que consistía fundamentalmente en memorizar muchos datos y hechos. Esta situación indeseable tenía, a su juicio, consecuencias negativas para España porque entendía que la historia patria era clave para el desarrollo presente de la nación, ya que el pasado ofrecía enseñanzas sobre qué hacer y qué no para llevar al país al éxito: «si el problema nacional estaba en la Escuela -como afirmaba el gran Costa-, dentro de la Escuela, el problema de la supervivencia de España, está en el modo de enseñar la Historia ${ }^{67}$. En su opinión, era importante trasladar a los niños las enseñanzas del heroísmo español, pero no solo el militar, sino el de todas las personas que en distintos ámbitos dieron gloria a España: los santos, los teólogos, los legisladores, los literatos o los científicos. Por este motivo, criticaba que la forma de escribir y explicar la historia se hubiese centrado en exceso en la historia política y militar, así como en las biografías de las grandes figuras, puesto que olvidaba las manifestaciones de la vida artística, científica, social o familiar.

Magdalena Santiago Fuentes puso estos planteamientos en práctica en su principal publicación, un compendio de la historia de la civilización, del que solo llegaron a publicarse los dos primeros volúmenes, que debía servir como un manual para la enseñanza que sintetizase las principales obras de los especialistas ${ }^{68}$. En el compendio analizó una amplia variedad de aspectos de las sociedades que abordó, como la cultura, las costumbres religiosas, las formas

66. El Eco Toledano, 14-4-1917, 3. Algunas historiadoras de prestigio han investigado sobre ella, pero no han prestado especial atención a su labor como historiadora: C. Muñoz Olivares, Los rincones de la vida: mujeres comprometidas: Magdalena de Santiago-Fuentes, Cuenca, 83-93; C. Flecha, "Magdalena de Santiago Fuentes Soto: 'Enseñar a leer es encender fuego', en VVAA, Treinta retratos de maestras: de la Segunda República hasta nuestros días, Madrid, 2005, 37-41; S. Hibbs-Lissorgues, "Magdalena de Santiago Fuentes (1873-1922) una obra polifacética al servicio del humanismo y del regeneracionismo", en F. Lafarga y L. Pegenaute (coords.), Autores traductores en la España del siglo XIX, Kassel, 2016, 573-582.

67. El Eco Toledano, 4, 5 y 6-6-1917, 3.

68. M. de Santiago Fuentes, Compendio de Historia de la Civilización, Tomo I. Edad Antigua, Madrid, 1911; M. de Santiago Fuentes, Compendio de Historia de la Civilización, Tomo II. Edad Media, Madrid, 1912. Algo similar hizo una maestra auxiliar de la Escuela Normal de Maestra de Valencia: C. Cervera Torres, Curso de Historia de España, Valencia, 1894. Aunque no todos los manuales escritos por españolas en el periodo incluyeron análisis detallados de la situación de las mujeres en el pasado: J. Saiz y Sánchez y J. F. Sánchez-Morate Martínez, Breves nociones de Geografía é Historia de España. Para uso de las aspirantes al título de Maestra de primera enseñanza elemental, Toledo, 1900; F. Bohigas, Actuación española en América. (Resumen escolar), Madrid, 1930. Que hubiese estas diferencias entre los trabajos de las historiadoras, independientemente de que tuviesen un perfil más profesionalizado o amateur, entra dentro de lo normal, pues hubo una buena cantidad de este tipo de obras escritos por españolas. Por señalar otros ejemplos: C. Calderón, Apuntes de Historia de España, Madrid, 1904; B. E. Martín González, Breves apuntes de Historia contemporánea de España y otras naciones, Madrid, 1919; B. E. Martín González, Nociones generales de Historia é Historia de la Edad antigua, Santiago, 1916; M. López Alcayde, Historia de España, Valencia, 1928. También antes de la guerra civil publicó el primero de sus múltiples manuales de historia María Comas: M. Comas, El mapa político de España a través de la historia, Barcelona, 1930. Asimismo, también publicó un trabajo de historia local: M. Comas, Juan López de Lazarraga. Secretario de los Reyes Católicos y el Monas- 
de gobierno, etc. En especial, destaca que prestase atención pormenorizada a la situación de las mujeres, sobre todo dentro del matrimonio, aunque no fuese un elemento predominante de su análisis. Por ejemplo, aseguró que

Únicamente el Cristianismo concedió á la mujer el derecho á la fidelidad del hombre dentro del matrimonio. La inferioridad social de la mujer se patentiza en la tutela ejercida sobre ella por el sexo fuerte, teniendo que redimirse de dicha tutela por dinero, como de una servidumbre. La mujer no podía heredar bienes inmuebles mientras existiere un varón, aunque fuera pariente más lejano, estando encomendados á ella los más rudos trabajos domésticos ó agrícolas ${ }^{69}$.

Su amplia dedicación a la creación de manuales de historia pensados para hacer la vida más fácil al estudiantado tiene que ver con su profesión, pero también con su formación, ya que en el CEH formó parte de algunos proyectos liderados por Rafael Altamira que tenían el objetivo de renovar los métodos didácticos de la enseñanza de la disciplina ${ }^{70}$. Por tanto, formó parte del reducido grupo de alumnas que pertenecieron al CEH antes de 1930, donde destacó junto a otras mujeres como María Concepción Alfaya López, Ángela Carnicer Pascual, Matilde Huici o María de Maeztu y Whitney ${ }^{71}$.

Asimismo, en sus estudios sobre el pasado, Magdalena Santiago Fuentes siempre prestó atención a múltiples aspectos y renegó de las explicaciones mono-causales. Esta tendencia, aparte de en el compendio reseñado, también queda patente en los seis libros que escribió sobre la historia y geografía de la mayoría de las regiones andaluzas. Bajo el genérico título de Geografía é Historia de esta provincia: montañas y ríos, pueblos, ciudades; sus monumentos, usos y costumbres; sus hijos ilustres (1911), abordó la historia de esos territorios hasta prácticamente el presente y, en la sección de personajes ilustres, también incluyó a algunas de las mujeres más destacadas, tanto del pasado lejano como del cercano ${ }^{72}$.

Un sistema similar siguió para su compendio de la historia española Luciana Casilda Monreal. De esta forma explicaba, por ejemplo, el papel de las mujeres durante la Edad Moderna:

terio de Bidaurreta en la villa de Oñate, Barcelona, 1936. Ver también la obra de Carmela Eulate titulada Isabel la Católica, su vida. Sus grandes obras narradas a los niños, Barcelona, 1935.

69. M. de Santiago Fuentes, Compendio de Historia de la Civilización, Tomo II. Edad Media, Madrid, $1912,89$.

70. López, Las ciencias sociales... op. cit., 126.

71. López, Las ciencias sociales... op. cit., 711-715.

72. M. de Santiago Fuentes, Almería: Geografía é Historia de esta provincia: montañas y ríos, pueblos, ciudades; sus monumentos, usos y costumbres; sus hijos ilustres, Barcelona, 1911; M. de Santiago Fuentes, Cádiz: Geografía é Historia de esta provincia: montañas y ríos, pueblos, ciudades; sus monumentos, usos y costumbres; sus hijos ilustres, Barcelona, 1911; M. de Santiago Fuentes, Córdoba: Geografía é Historia de esta provincia: montañas y ríos, pueblos, ciudades; sus monumentos, usos y costumbres; sus hijos ilustres, Barcelona, 1911; M. de Santiago Fuentes, Huelva: Geografía é Historia de esta provincia: montañas y ríos, pueblos, ciudades; sus monumentos, usos y costumbres; sus hijos ilustres, Barcelona, 1911; M. de Santiago Fuentes, Jaén: Geografía é Historia de esta provincia: montañas y ríos, pueblos, ciudades; sus monumentos, usos y costumbres; sus hijos ilustres, Barcelona, 1911; M. de Santiago Fuentes, Sevilla: Geografía é Historia de esta provincia: montañas y ríos, pueblos, ciudades; sus monumentos, usos y costumbres; sus hijos ilustres, Barcelona, 1911. 
En época de tanta cultura intelectual, la mujer debía tomar una parte importante en todas las manifestaciones de la vida, siendo respetada en medio de los ejércitos, como sucedió con la hija del Gran Capitán, que acompañó á su padre en las guerras de Italia. Cuando se hacía la primera enseñanza obligatoria y se incapacitaba para ser alcaldes á los que no sabían leer y escribir, no es de extrañar que las mujeres tomaran una parte activa en las Academias y explicaran en las Universidades, cuyo número aumentó considerablemente hasta Felipe $\mathrm{II}^{73}$.

Como el que hubiese españolas como profesoras en las universidades en aquel periodo fue un aspecto que llamó la atención en el extranjero, algunas historiadoras foráneas acudieron a España para investigar sobre el tema. Por ejemplo, la alemana Therese Oettel trabajó acerca de la figura de Lucía de Madrano. Sobre esta mujer, en 1935 publicó un meticuloso trabajo en el que ofrecía datos que podrían respaldar que llegase a ser catedrática en tiempos de Isabel la Católica, a la vez que presentó otros que lo pondrían en cuestión. En cualquier caso, aseguraba que era sorprendente la posición académica tan relevante de esa mujer en el siglo XVI, cuando en el XX todavía había un reducido número de mujeres estudiantes en las universidades españolas. Para intentar dar explicación a la situación, aseguró que «no pudo ser debido más que a la influencia, en gran parte, de aquella mujer extraordinaria que se llamó Isabel $\mathrm{I} »^{74}$.

Asimismo, en este tipo de manuales generalistas, mujeres como Ana Arizmendi (1897) prestaron especial atención al vestido de las mujeres en el pasado:

El talle que aparece en el traje de la mujer en el siglo XV, concluyó por estrecharse en el XVII de un modo absurdo por medio del corsé; cuyo uso antihigiénico aún impera, con detrimento del buen sentido; de la salud de la mujer y de sus hijos, y aún de la estética; pues reducir el talle á las dimensiones del cuello, equivale á destruir la verdadera belleza que consiste en las proporciones armónicas del cuerpo ${ }^{75}$.

Una apreciación que, sin duda, estaba marcada por el contexto finisecular en el que la autora escribió la obra, pues la crítica del corsé por parte de los sectores higienistas, a nivel transnacional, estaba calando en el conjunto de la sociedad ${ }^{76}$.

Por último, los años finales de la Segunda República y los primeros de la dictadura franquista fueron una época fructífera para el trabajo histórico de las mujeres de las culturas políticas de extrema derecha. De entre todas, destaca una obra de Mercedes Sanz Bachiller compuesta por 37 pequeñas biografías de algunas de las mujeres más destacadas de la historia de España (1940), como Doña Urraca, María de Molina, Isabel la Católica, María de Portugal, Santa Teresa de Jesús, Agustina de Aragón, Cecilia Böhl de Faber, Concepción Arenal,

73. L. C. Monreal, Compendio de la historia de España, Barcelona, 1898, 229-231.

74. T. Oettel, "Una catedrática en el siglo de Isabel la Católica: Luisa (Lucía) de Medrano”, Boletín de la Real Academia de la Historia, 107, 1935, 290.

75. A. Arizmendi de Sanz, Elementos de historia de España, Sevilla, 1897, 114.

76. M. L. Fernández, La imagen de la mujer en la pintura española. 1890-1914, Madrid, 2006, 57-60, 123 127. 
Rosalía de Castro, Emilia Pardo Bazán, Concepción Gimeno de Flaquer o Sofía Casanova. Independientemente del campo en el que se habían destacado, la autora resaltó sus méritos (que lograban sin desatender las labores que Sanz Bachiller consideraba como propias de su sexo), su relación con el poder, sus acciones caritativas, su espíritu religioso y su sentimiento patriótico ${ }^{77}$. No obstante, también hubo otras publicaciones históricas más generalistas, como un artículo de la falangista María del Rosario de Nárdiz en el que narró la patriótica historia de un regimiento de españoles destinado en Dinamarca que, en 1808, actuó para liberar a España de Napoleón ${ }^{78}$.

\section{Conclusiones}

Durante las últimas décadas del siglo XIX y las primeras del XX, las mujeres españolas, al igual que ocurrió en la mayoría del mundo, fueron relegadas, explícita o implícitamente, a los márgenes de la profesión de historiador. Sin embargo, las historiadoras semiprofesionales o aficionadas aprovecharon que tanto el análisis del periodo contemporáneo como la erudición localista, centrada principalmente en heráldica, genealogías y biografías, fueron considerados dentro de la historiografía profesional como ámbitos de poco valor, para investigar en estos campos con una libertad temática que no habrían tenido en otros. Como el número de historiadoras profesionales antes de la guerra civil española fue muy reducido, la mayoría de la producción historiográfica de autoría femenina en España debe buscarse entre las historiadoras aficionadas y semiprofesionales, lo cual fue una situación similar a la que experimentaron las mujeres de los otros países europeos y americanos. En cualquier caso, independientemente de que se las pueda considerar como historiadoras semiprofesionales o aficionadas, las mujeres que escribieron sobre el pasado intentaron acercar su forma de investigar al positivismo metodológico, por lo que durante el primer tercio del siglo XX sus publicaciones no se diferenciaron mucho de los trabajos de los historiadores profesionales. Además, en sus estudios se preocuparon por la construcción nacionalista de la historia, el aspecto más valorado por los historiadores profesionales del periodo, pues todos tuvieron una agenda específica detrás de sus investigaciones sobre el pasado.

Las historiadoras católicas semiprofesionales y aficionadas buscaron colaborar mediante sus trabajos en el proceso de la construcción de la nación española, a la vez que trataron de construir su identidad como mujeres en relación con la propia nación y su pasado, pues hubo una compleja pero estrecha relación entre género, nación, catolicismo e historia/historiografía. En concreto, estas historiadoras establecieron una relación de descendencia directa

77. M. Sanz Bachiller, Mujeres de España, Madrid, 1940. También la carlista María Rosa Urraca Pastor escribió varias biografías en estos años, destacando la de San Francisco de Borja, que aprovechó para exaltar la historia imperial de España y la reconquista, y la de una actriz llamada Lola Montes, que debía servir como un ejemplo a no seguir para sus lectoras: M. R. Urraca Pastor, San Francisco de Borja, Barcelona, 1943, 7-17 y 138; M. R. Urraca Pastor, Lola Montes, Barcelona, s/f.

78. Historia. Revista de estudiantes, 5-1935, 131-139; Pensamiento alavés, 9-8-1938, 3. 
entre las católicas españolas del presente y las españolas más destacadas del pasado, especialmente con aquellas que habían realizado alguna gran hazaña en defensa de la religión o de la patria. El motivo es que estas historiadoras católicas buscaban justificar y legitimar con argumentos históricos la capacidad de las mujeres del presente para intervenir en la sociedad y para movilizarse por la patria y, a la vez, pretendían moralizar a sus lectoras presentándolas ejemplos a seguir. No obstante, si bien el objetivo de estas historiadoras semiprofesionales o aficionadas fue crear una conexión entre el pasado y el presente, estos trabajos normalmente fueron fruto de investigaciones rigurosas que se rigieron, a rasgos generales, por los métodos y cánones demandados por la academia de la época.

Durante el periodo analizado en la presente investigación, los estudios realizados por historiadoras católicas semiprofesionales o aficionadas centrados en la historia de las mujeres españolas se aliaron con el enfoque biográfico. La tendencia general de estos trabajos era, por un lado, intentar que las lectoras tuviesen referentes moralizantes e inspiradores en los que fijarse y, por otro lado, denunciar los obstáculos adicionales que en su vida encontraban las mujeres, tanto del pasado como del presente, por el hecho de ser mujeres. Por tanto, fueron muchas las historiadoras semiprofesionales y aficionadas que decidieron investigar de forma específica sobre las mujeres del pasado e incluirlas en sus estudios generalistas de historia, ya fuese española o universal. Tanto fue así que las mujeres tuvieron más presencia en los estudios históricos a finales del siglo XIX y el primer tercio del XX que en las décadas de los cuarenta, cincuenta o sesenta. 